Check for updates

Cite this: RSC Adv., 2018, 8, 13675

Received 26th January 2018

Accepted 2nd April 2018

DOI: $10.1039 / \mathrm{c} 8 \mathrm{ra00799c}$

rsc.li/rsc-advances

\title{
Tuning the structure and properties of a multiferroic metal-organic-framework via growing under high magnetic fields $\uparrow$
}

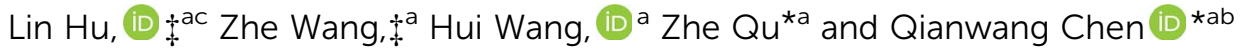

High magnetic field-induced synthesis has been demonstrated to tune the structure and properties of the multiferroic metal-organic framework $\left[\left(\mathrm{CH}_{3}\right)_{2} \mathrm{NH}_{2}\right]\left[\mathrm{Mn}(\mathrm{HCOO})_{3}\right]$. The crystals obtained under $90 \mathrm{kOe}$ exhibit a lower ferroelectric polarization value and reduced magnetic susceptibility, compared to crystals obtained without a field, which is attributed to structural changes induced by the high magnetic field.

In 2009, Jain et al. reported multiferroic behavior in a family of perovskite metal-organic frameworks (MOFs), which stimulated considerable experimental and theoretical efforts to search for new multiferroic materials among perovskite MOFs. ${ }^{1}$ In particular, MOFs with the $\mathrm{ABX}_{3}$ perovskite-like structure are of great interest because the variable $\mathrm{A}$ and $\mathrm{B}$ components provide plenty of room for adjusting the physical and chemical properties in a simple crystalline structure. ${ }^{2-25}$ One such example is formate-based perovskites, $\left[\mathrm{A}-\mathrm{M}-(\mathrm{HCOO})_{3}\right]$. They contain a divalent transition metal ion $\left(\mathrm{M}^{2+}\right)$ which occupies the center of a $\mathrm{BO}_{6}$ octahedron, linked by a formate $\left(\mathrm{HCOO}^{-}\right)$ bridging ligand to form a cavity which is occupied by an organic cation. The ordering of hydrogen bonding $(\mathrm{N}-\mathrm{H} \cdots \mathrm{O})$ of the alkylammonium cation triggers the ferroelectic ordering at low temperature, while the weak ferromagnetism arises from metal ions.

It is well known that volatilization and hydrothermal/ solvothermal synthesis are the main methods to grow formate-based perovskites, which involve nucleation and growth processes that are affected by physical and chemical factors. Modifying or controlling the physical environment is a simple means to affect the processes of crystallization in order to fine-tune the crystal structure and properties. On the other

\footnotetext{
${ }^{a}$ Anhui Province Key Laboratory of Condensed Matter Physics at Extreme Conditions, High Magnetic Field Laboratory, Chinese Academy of Sciences, Hefei 230031, Anhui, China. E-mail: zhequ@hmfl.ac.cn

${ }^{b}$ Hefei National Laboratory for Physical Science at Microscale, Department of Materials Science \& Engineering, Collaborative Center of Suzhou Nano Science and Technology, University of Science and Technology of China, Hefei 230026, China. E-mail: cqw@ustc.edu.cn

'Key Laboratory of Photovoltaic and Energy Conservation Materials, Institute of Applied Technology, Hefei Institutes of Physical Science, Chinese Academy of Sciences, Hefei 230031, China

$\dagger$ Electronic supplementary information (ESI) available: Detailed experimental procedures; XRD patterns and illustration of experimental apparatus. See DOI: $10.1039 / \mathrm{c} 8 \mathrm{ra00799c}$

‡. H. and Z. W contributed equally to this work.
}

hand, the effect of pressure on the structure modification of multiferroic MOFs has recently been demonstrated, which showed that ferroelectric polarization could be enhanced by more than three times by applying a compressive strain to the multiferroic frameworks. ${ }^{14}$ Also, density functional theory calculations indicated that changing the magnitude or the canting of the organic molecular dipole can tune the ferroelectric polarization in the new class of multiferroic metalorganic frameworks. ${ }^{4,15}$ These experimental results further highlight the potential of these flexible MOF perovskites to undergo large structural changes in response to an external stimulus.

Crystal growth under a magnetic field is an interesting research topic because a magnetic field may provide a special environment. From the latter half of the 1990s, many studies of magnetic field effects (MFEs) on protein crystallization have been carried out. ${ }^{26-28}$ It has been found that magnetic fields can induce molecular ordering in most organic polymer and biological macromolecules due to the magnetic susceptibility anisotropy of the individual $\mathrm{C}-\mathrm{C}, \mathrm{C}-\mathrm{O}, \mathrm{C}-\mathrm{H}$, and $\mathrm{O}-\mathrm{H}$ bonds and their relative orientation in the crystal, or the formation of an interlinked network of biological macromolecules under a high magnetic field. ${ }^{29-31}$ Also, the MFEs on the growth of materials have been extensively studied by our group, which found that both the structure and the properties of materials can be regulated by magnetic fields. ${ }^{32-38}$ For example, it is believed that during $\mathrm{Co}_{3} \mathrm{O}_{4}$ growth, the alignment of the spins and thus the magnetic and crystal lattices of $\mathrm{Co}_{3} \mathrm{O}_{4}$ are influenced by the external magnetic field. ${ }^{35}$ In this paper, we extend the study to grow single crystals of formate-based perovskites $\left[\left(\mathrm{CH}_{3}\right)_{2} \mathrm{NH}_{2}\right]\left[\mathrm{Mn}(\mathrm{HCOO})_{3}\right](\mathrm{DMMnF})$ under a high magnetic field. DMMnF is the first prototypical multiferroic formatebased perovskite to be discovered ${ }^{1}$ which exhibits an orderdisorder ferroelectric transition below $185 \mathrm{~K}$. It is also a weakly canted antiferromagnet $\left(T_{\mathrm{c}}=8.5 \mathrm{~K}\right)$ with a $0.08^{\circ}$ canting angle. Moreover, DMMnF is suggested to be 
thermodynamically more stable than other formate-based perovskites and is easily obtained by a solvothermal process. The main purpose of this study is to show the possibility that the structure and properties of multiferroic MOFs could be tuned by synthesis under high magnetic field, and to try to understand the mechanism. In this work, the growth of DMMnF crystals was carried out with and without an applied magnetic field. The resulting crystals were labeled as AFDMMnF (applied field) and ZF-DMMnF (zero field), with AFDMMnF indicating the product obtained under a high magnetic field, and ZF-DMMnF indicating the case without a magnetic field.

The AF-DMMnF and ZF-DMMnF micro-crystal powders (obtained from grinding the as-grown single crystals) and single crystals were characterized by X-ray diffraction (XRD). As shown in Fig. S2a, $\uparrow$ the clear sharp peaks of both samples are in good agreement with the theoretical patterns calculated from the single-crystal data. ${ }^{15}$ Moreover, the peaks of the powder samples could be attributed to the diffraction planes of the trigonal phase DMMnF at room temperature, confirming the phase purity of both samples (Fig. S2b $\dagger$ ).

Dielectric constant measurements were carried out on single crystals of AF-DMMnF and ZF-DMMnF. The dielectric constants of both samples show a clear anomaly close to $185 \mathrm{~K}$ on cooling (Fig. 1a). The shape of the dielectric plot indicates that the samples are undergoing a paraelectric to ferroelectric phase transition, which is consistent with the literature..$^{\mathbf{1 1 6}}$ It should be noted that the dielectric constant of AF-DMMnF is lower than that of ZF-DMMnF above $185 \mathrm{~K}$. Fig. 1b shows the temperature dependence of the electric polarization. Below the phase transition temperature, the electric polarization values of AFDMMnF are much lower than those of ZF-DMMnF, suggesting that the electric polarization of DMMnF in the ferroelectric state is reduced by growing under a high magnetic field. The highest experimental electric polarizations of AF-DMMnF and ZFDMMnF within the measured temperature range are $\sim 0.3 \mu \mathrm{C}$ $\mathrm{cm}^{-2}$ and $\sim 1 \mu \mathrm{C} \mathrm{cm}^{-2}$, respectively, as shown in Fig. 1b. It is well known that the ferroelectrics of DMMnF arise from the hydrogen bonds between the $\mathrm{DMA}^{+}$cations and the formate framework. The reduction in electric polarization indicates that the hydrogen bond-related interactions may have changed. As is known, the Raman spectrum is sensitive to changes in chemical
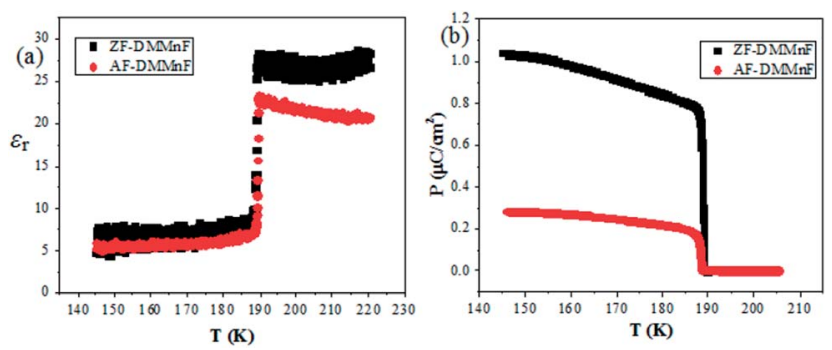

Fig. 1 (a) Dielectric constants of AF-DMMnF and ZF-DMMnF as a function of temperature at $1 \mathrm{kHz}$; (b) the electric polarization of AF$\mathrm{DMMnF}$ and ZF-DMMnF as a function of temperature. Electric polarization was measured after cooling the sample at a voltage of $750 \mathrm{~V} \mathrm{~cm}^{-1}$. bonding. The Raman spectra of the samples at different temperatures are presented in Fig. 2, and the main vibration modes assigned to $\mathrm{HCOO}^{-}$and $\mathrm{DMA}^{+}$have been marked. All bands observed below $300 \mathrm{~cm}^{-1}$ can be attributed to the lattice modes (Fig. 2a). It is worth noting that the intensities of $\nu_{1}\left(\mathrm{HCOO}^{-}\right), \nu_{2}\left(\mathrm{HCOO}^{-}\right)$and $\nu_{3}\left(\mathrm{HCOO}^{-}\right)$are weakened in $\mathrm{AF}-$ DMMnF compared to those in ZF-DMMnF. In contrast, the intensity of $\nu_{\mathrm{s}}(\mathrm{CNC})$ at $897 \mathrm{~cm}^{-1}$ in AF-DMMnF is increased compared to that in ZF-DMMnF. The obvious weakening in the vibrational modes of the formate ions in AF-DMMnF could presumably be influenced by the strength of the $\mathrm{N}-\mathrm{H} \cdots \mathrm{O}$ hydrogen bonds between the $\mathrm{DMA}^{+}$cations and the formate framework..$^{15}$ It is suggested that the band at $2788 \mathrm{~cm}^{-1}$ can be unambiguously assigned to the stretching modes of the $\mathrm{NH}_{2}$ group which directly involve hydrogen-bonds. ${ }^{12}$ Most importantly, at $140 \mathrm{~K}$, the intensity of $\nu\left(\mathrm{NH}_{2}\right)$ in AF-DMMnF is much lower than that in ZF-DMMnF, as shown in Fig. 2d. This result, combined with the weakened vibration modes of $\mathrm{HCOO}^{-}$, indicates the reduced strength of the hydrogen-bonds in AFDMMnF, which is responsible for the decrease in polarization values. Several claims have been made that magnetic fields change the physicochemical properties of water or prepared laboratory solutions, by influencing nucleation and growth, chemical equilibria, and so on. ${ }^{39-41}$ An enhancement in the hydrogen-bonded strength under a magnetic field of $100 \mathrm{kOe}$ was observed, which was caused by increased electron delocalization in the hydrogen-bonded molecules. ${ }^{\mathbf{4 2 , 4 3}}$ Our case can be considered as an example of MFEs on a hydrogen-bonded structure, although the mechanism has as yet not been adequately explained. Fig. $\mathrm{S} 3 \dagger$ is the optical image of the AFDMMnF and ZF-DMMnF surfaces. The AF-DMMnF exhibits uniform growth steps, which may be attributed to slower nucleation. However, irregular growth layers are observed on the surfaces of the ZF-DMMnF because of rapid nucleation. ${ }^{15}$
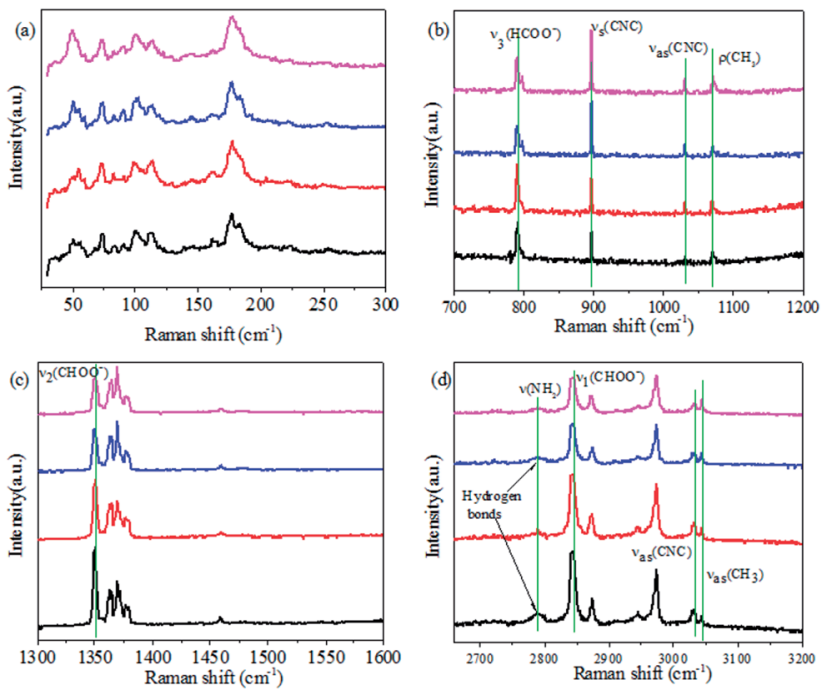

Fig. 2 Detail of the Raman spectra corresponding to the spectral ranges 25-300, 700-1200, 1300-1600 and 2650-3200 $\mathrm{cm}^{-1}$ at different temperatures for the samples. Black lines: $140 \mathrm{~K}$ for ZFDMMnF; red line: $170 \mathrm{~K}$ for ZF-DMMnF; blue line: $140 \mathrm{~K}$ for AF-DMMnF; pink line: $170 \mathrm{~K}$ for $\mathrm{AF}-\mathrm{DMMnF}$. 
Wang et al. have shown that DMMnF is a canted weak ferromagnet with a $T_{\mathrm{c}}$ value of $8.5 \mathrm{~K} .{ }^{44}$ It is suggested that the spin canting may originate from the noncentrosymmetric character of the three-atom formate bridge, $\mathrm{CHOO}^{-}$. Fig. 3a and $\mathrm{b}$ show the magnetization as a function of temperature measured at 200 Oe. Both samples show a clear magnetic phase transition at $c a .8 .5 \mathrm{~K}$. Furthermore, the isothermal magnetization $M(T, H)$ at $1.8 \mathrm{~K}$ is shown in Fig. 3c. The magnetization increases almost linearly from 0 to $10 \mathrm{kOe}$. It is worth mentioning that the magnetic susceptibilities of AF-DMMnF are lower than those of ZF-DMMnF. In the low-field range at $1.8 \mathrm{~K}$, a hysteresis loop can be observed with remnant magnetizations $M_{\mathrm{R}}=0.0069 \mu \mathrm{B}$ and $0.0045 \mu \mathrm{B}$ for ZF-DMMnF and AF-DMMnF, respectively. The canting angle $\alpha$ is related to $M_{\mathrm{R}}$ and $M_{\mathrm{S}}$ through $\sin (\alpha)=M_{\mathrm{R}} / M_{\mathrm{S}}\left(M_{\mathrm{S}}=5 \mu \mathrm{B}\right.$ for a spin-only $\mathrm{Mn}^{\mathrm{II}}$ ion $)$. In ZF-DMMnF, the canting angle $\alpha$ is estimated to be about $0.08^{\circ}$, which is consistent with the reported result. ${ }^{44}$ However, the canting angle $\alpha$ in AF-DMMnF is reduced to $0.05^{\circ}$ according to the above formula. In a canted antiferromagnetic material, neighboring spins do not align in a strictly parallel manner, but cant each other at a certain angle. During the growth process, the applied magnetic field may disturb this manner and change the angles between the directions of neighboring spins. The decrease in canting angle in AF-DMMnF will generate fewer uncompensated spins, leading to reduced magnetization. This result proves that growth under a magnetic field may be used to control the degree of spin-canting, and then influence the magnetic properties.

To further investigate the magnetic states in ZF-DMMnF and AF-DMMnF below $T_{\mathrm{c}}$, a low temperature electron spin resonance (ESR) technique is employed. ESR embodies the internal environment (crystal field and internal magnetic field) of magnetic ions. Both samples possess a temperatureindependent peak and another peak which moves to a low
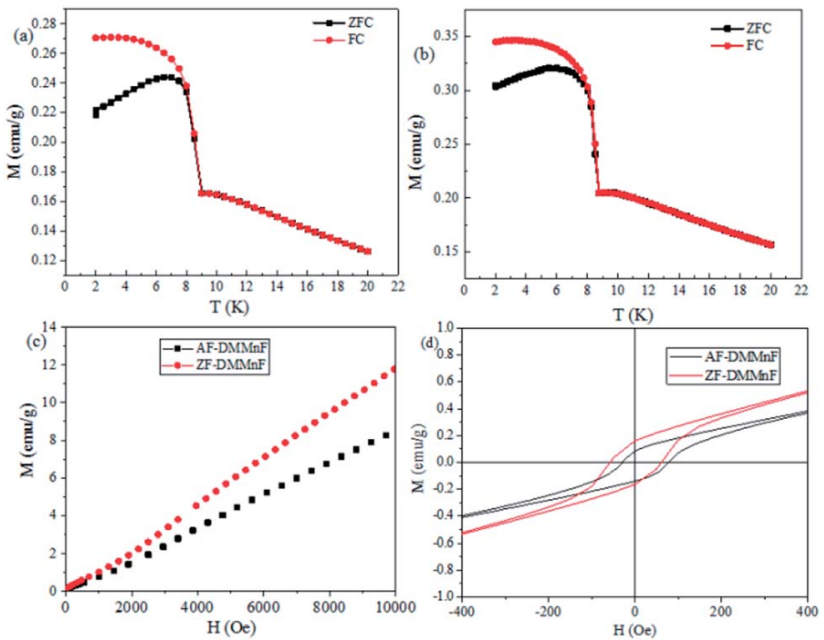

Fig. 3 Temperature dependence of $\chi_{M}$ of AF-DMMnF (a) and ZF$\mathrm{DMMnF}$ (b) measured at 200 Oe from 2 to $20 \mathrm{~K}$ (ZFC and FC). (c) Fielddependent isothermal magnetization $M(T, H)$ for $\mathrm{AF}-\mathrm{DMMnF}$ and $\mathrm{ZF}$ $\mathrm{DMMnF}$ at $1.8 \mathrm{~K}$ from 0 to $10 \mathrm{kOe}$. (d) The hysteresis loop measured at $1.8 \mathrm{~K}$.
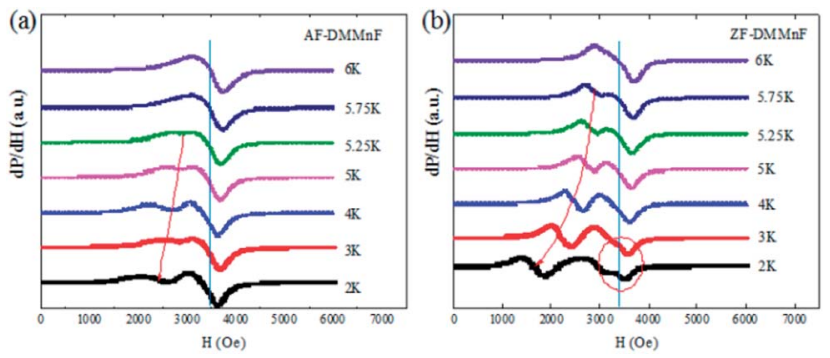

Fig. 4 The ESR spectra of AF-DMMnF (a) and ZF-DMMnF (b) at selected temperatures.

magnetic field when the temperature decreases (Fig. 4). The temperature-independent peak comes from the transition between the Zeeman energy levels of $S= \pm 1 / 2$, and the low-field peaks are derived from the transition between the Zeeman energy level of $S=1 / 2$ and that of $S=3 / 2$ (zero splitting energy, $D<0)$ or $S=-1 / 2$ and $S=-3 / 2(D>0) .{ }^{45}$ Interestingly, the separation distances between the two peaks of AF-DMMnF are smaller than those of ZF-DMMnF (Fig. 4), indicating the increased zero splitting energies in AF-DMMnF. Moreover, additional weak peaks (circled) appear in the ESR spectrum of ZF-DMMnF, which are probably caused by a small number of magnetic domains in different directions. In other words, the magnetic field makes the magnetic domains of AF-DMMnF more consistent, which will contribute to the dielectric constants and electric polarization values. However, the slight contribution of the domains cannot compensate for the decreases in dielectric constant and electric polarization values induced by the reduction in strength of the hydrogen-bonds. Therefore, AF-DMMnF exhibits a reduced dielectric constant and electric polarization. The increased zero splitting energies, combined with more consistent magnetic domains, further proves that the structure of DMMnF can be regulated by growth under high magnetic fields.

In summary, for the first time, it has been demonstrated that the structure and properties of a multiferroic MOF can be tuned by growth under a high magnetic field. The crystals obtained under a high magnetic field exhibit a much lower electric polarization value, which is attributed to the reduced strength of the hydrogen bonds. Moreover, a decrease in magnetic susceptibilities and remnant magnetization was observed due to the reduction in the spin canting angle. With the emergence of superconducting technology, a high magnetic field could be obtained easily, which would open a possible way to tailor the structures and properties of multiferroic MOFs by high magnetic field-induced synthesis.

\section{Conflicts of interest}

There are no conflicts to declare.

\section{Acknowledgements}

We gratefully acknowledge financial support from the National Key R\&D Program of China (Grant No. 2016YFA0401801), the Hefei 
Science Center CAS (2016HSC-IU011), the Natural Science Foundation of China (Grant No. 21301178, U1532153 and 11774352), and the Major Program of Development Foundation of Hefei Center for Physical Science and Technology (Grant No. 2016FXZY001).

\section{Notes and references}

1 P. Jain, V. Ramachandran, R. J. Clark, H. D. Zhou, B. H. Toby, N. S. Dalal, H. W. Kroto and A. K. Cheetham, J. Am. Chem. Soc., 2009, 131, 13625-13627.

2 R. Ramesh, Nature, 2009, 461, 1218-1219.

3 G. C. Xu, W. Zhang, X. M. Ma, Y. H. Chen, L. Zhang, H. L. Cai, Z. M. Wang, R. G. Xiong and S. Gao, J. Am. Chem. Soc., 2011, 133, 14948-14951.

4 D. D. Sante, A. Stroppa, P. Jain and S. Picozzi, J. Am. Chem. Soc., 2013, 135, 18126-18130.

5 L. C. G. mez-Aguirre, B. Pato-Doldán, J. Mira, S. CastroGarcía, M. A. Seńarís-Rodríguez, M. Sánchez-Andújar, J. Singleton and V. S. Zapf, J. Am. Chem. Soc., 2016, 138, 1122-1125.

6 Y. Tian, S. P. Shen, J. Z. Cong, L. Q. Yan, S. G. Wang and Y. Sun, J. Am. Chem. Soc., 2016, 138, 782-785.

7 J. P. Zhao, J. Xu, S. D. Han, Q. L. Wang and X. H. Bu, Adv. Mater., 2017, 29, 1606966.

8 Y. Tian, W. Wang, Y. S. Chai, J. Z. Cong, S. P. Shen, L. Q. Yan, S. G. Wang, X. F. Han and Y. Sun, Phys. Rev. Lett., 2014, 112, 017202.

9 F. R. Fan, H. Wu, D. Nabok, S. Hu, W. Ren, C. Draxl and A. Stroppa, J. Am. Chem. Soc., 2017, 139, 12883-12886.

10 A. J. Clune, K. D. Hughey, C. Lee, N. Abhyankar, X. Ding, N. S. Dalal, M.-H. Whangbo, J. Singleton and J. L. Musfeldt, Phys. Rev. B, 2017, 96, 104424.

11 Y. Ma, J. Z. Cong, Y. S. Chai, L. Q. Yan, D. S Shang and Y. Sun, Appl. Phys. Lett., 2017, 111, 042901.

12 M. Maczzka, A. Gągor, B. Macalik, A. Pikul, M. Ptak and J. Hanuza, Inorg. Chem., 2014, 53, 457-467.

13 M. Maczka, T. A. Silva, W. Paraguassu, M. Ptak and K. Hermanowicz, Inorg. Chem., 2014, 53, 12650-12657.

14 S. Ghosh, D. D. Sante and A. Stroppa, J. Phys. Chem. Lett., 2015, 6, 4553-4559.

15 L. P. Xin, Z. Fan, G. H. Li, M. Zhang, Y. H. Han, J. Wang, K. P. Ong, L. Qin, Y. Z. Zheng and X. J. Lou, New J. Chem., 2017, 41, 151-159.

16 P. Jain, A. Stroppa, D. Nabok, A. Marino, A. Rubano, D. Paparo, M. Matsubara, H. Nakotte, M. Fiebig, S. Picozzi, E. S. Choi, A. K. Cheetham, C. Draxl, N. S. Dalal and V. S Zapf, npj Quantum Materials, 2016, 1, 16012.

17 W. P. Zhao, C. Shi, A. Stroppa, D. D. Sante, F. Cimpoesu and W. Zhang, Inorg. Chem., 2016, 55, 10337-10342.

18 M. E. Kamminga, A. Stroppa, S. Picozzi, M. Chislov, I. A. Zvereva, J. Baas, A. Meetsma, G. R. Blake and T. T. M. Palstra, Inorg. Chem., 2017, 56, 33-41.

19 L. Mazzuca, L. Cañadillas-Delgado, J. A. RodríguezVelamazán, O. Fabelo, M. Scarrozza, A. Stroppa, S. Picozzi, J. P. Zhao, X. H. Bu and J. Rodríguez-Carvajal, Inorg. Chem., 2017, 56, 197-207.
20 M. Ptak, M. Mączka, A. Gągor, A. Sieradzki, A. Stroppa, D. Di Sante, J. M. Perez-Matoe and L. Macalik, Dalton Trans., 2016, 45, 2574-2583.

21 A. Stroppa, D. D. Sante, P. Barone, M. Bokdam, G. Kresse, C. Franchini, M. H. Whangbo and S. Picozzi, Nat. Commun., 2014, 5, 5900, DOI: 10.1038/ncomms6900.

22 L. C. Gómez-Aguirre, B. Pato-Doldán, A. Stroppa, S. YáñezVilar, L. Bayarjargal, B. Winkler, S. Castro-García, J. Mira, M. Sánchez-Andújar and M. A. Señarís-Rodríguez, Inorg. Chem., 2015, 54, 2109-2116.

23 A. Stroppa, P. Jain, P. Barone, M. Marsman, J. M. Perez-Mato, A. K. Cheetham, H. W. Kroto and S. Picozzi, Angew. Chem., Int. Ed., 2011, 50, 5847-5850.

24 A. Stroppa, P. Barone, P. Jain, J. M. Perez-Mato and S. Picozzi, Adv. Mater., 2013, 25, 2284-2290.

25 Y. Tian, A. Stroppa, Y. S. Chai, P. Barone, M. Perez-Mato, S. Picozzi and Y. Sun, Phys. Status Solidi RRL, 2015, 9, 62-67.

26 E. K. Yan, C. Y. Zhang, J. He and D. C. Yin, Int. J. Mol. Sci., 2016, 17, 1906.

27 A. R. Romero, N. E. Escofet, C. P. Rivera and A. Moreno, Crystals, 2017, 7, 179, DOI: 10.3390/cryst7060179.

28 L. J. Huang, H. L. Cao, Y. J. Ye, Y. M. Liu, C. Y. Zhang, Q. Q. Lu, H. Hou, P. Shang and D. C. Yin, CrystEngComm, 2015, 17, 1237-1241.

29 J. Sugiyama, H. Chanzy and G. Maret, Macromolecules, 1992, 25, 4232-4234.

30 X. M. Dong and D. G. Gray, Langmuir, 1997, 13, 2404-2409. 31 H. Bi, Q. W. Chen, Y. L. Zhuang and S. Q. Zhao, J. Magn. Magn. Mater., 2006, 307, 38-42.

32 J. Wang, Q. W. Chen, C. Zeng and B. Y. Hou, Adv. Mater., 2004, 16, 137-140.

33 L. X. Sun, Q. W. Chen, Y. Tang and Y. Xiong, Chem. Commun., 2007, 27, 2844-2846.

34 M. S. Wang and Q. W. Chen, Chem. - Eur. J., 2010, 16, 1208812090.

35 M. S. Wang, L. K. Zeng and Q. W. Chen, Dalton Trans., 2011, 40, 597-601.

36 L. Hu, R. R. Zhang and Q. W. Chen, Nanoscale, 2014, 6, 14064-14105.

37 L. Hu, X. Y. Feng, L. Z. Wei, K. J. Zhang, J. M. Dai, Y. C. Wu and Q. W. Chen, Nanoscale, 2015, 7, 10925-10930.

38 W. Ding, L. Hu, Z. G. Sheng, J. M. Dai, X. B. Zhu, X. W. Tang, Z. Z. Hui and Y. P. Sun, CrystEngComm, 2016, 18, 6134-6137.

39 R. Cai, H. W. Yang, J. S. He and W. P. Zhu, J. Mol. Struct., 2009, 938, 15-19.

40 M. Morimitsu, K. Shiomi and M. Matsunaga, J. Colloid Interface Sci., 2000, 229, 641-643.

41 L. Holysz, M. Chibowski and E. Chibowski, Colloids Surf., A, 2002, 208, 231-240.

42 K. T. Chang and C. I. Weng, J. Appl. Phys., 2006, 100, 043917.

43 H. Hosoda, H. Mori, N. Sogoshi, A. Nagasawa and S. Nakabayashi, J. Phys. Chem. A, 2004, 108, 1461-1464.

44 X. Y. Wang, L. Gan, S. W. Zhang and S. Gao, Inorg. Chem., 2004, 43, 4615-4625.

45 S. Isber and M. Averous, Phys. Rev. B, 1995, 51, 15211. 